Eppur si muove: pensiero e pratiche di genere, tra accademia, mondo associativo ed editoriale femminista

"Eppur si muove» : pensée et pratique de genre, entre académie, monde associatif et éditorial féministe

Nadia Setti

(2) OpenEdition

Journals

Edizione digitale

URL: http://journals.openedition.org/cei/1065

DOI: $10.4000 /$ cei. 1065

ISSN: 2260-779X

Editore

UGA Éditions/Université Grenoble Alpes

Edizione cartacea

Data di pubblicazione: 30 juin 2013

Paginazione: $39-49$

ISBN: 978-2-84310-245-5

ISSN: 1770-9571

Notizia bibliografica digitale

Nadia Setti, «Eppur si muove: pensiero e pratiche di genere, tra accademia, mondo associativo ed editoriale femminista», Cahiers d'études italiennes [Online], 16 | 2013, online dal 15 décembre 2014 consultato il 26 mars 2021. URL: http://journals.openedition.org/cei/1065 ; DOI: https://doi.org/ 10.4000/cei.1065 


\title{
EPPUR SI MUOVE: PENSIERO E PRATICHE DI GENERE, TRA ACCADEMIA, MONDO ASSOCIATIVO ED EDITORIALE FEMMINISTA
}

\author{
Nadia Setti \\ Université Paris 8
}

Un resoconto di Veronica Pravadelli sul convegno organizzato all'università di Roma 3 da Paola Bono, nel marzo 2009, sullo stato degli studi di genere nelle università italiane ed europee, ha suscitato un dibattito sulla

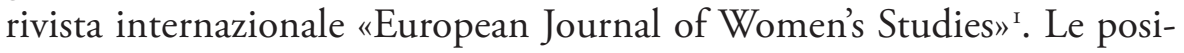
zioni contrastanti riguardavano la collocazione ed estensione degli studi di genere nelle università e istituti di ricerca italiani ${ }^{2}$. Rinvio agli articoli citati per il dettaglio degli argomenti sviluppati, ma si può certo sostenere che la realtà italiana è forse in parte più complessa, meno leggibile

I. Si tratta del convegno Gendering the Academy. Italian Experiences and Experiments. Un altro genere di università. Esperienze e sperimentazioni (cfr. <www.genderingtheacademy.jimdo.com>, data di ultima consultazione 8 dicembre 20I2). Gli articoli pubblicati sono di Pravadelli, nella sezione Open Forum, Women and Gender Studies, Italian Style, in «European Journal of Women's Studies», I7, I, February 2010, pp. 61-67, e di Chiara Saraceno, sempre nella rubrica Open Forum, Women and gender Studies in Italy: Lack of Institutionalisation or a Different Kind of Institutionalisation?, in «European Journal of Women's Studies», 17, 3, August 20Io, pp. 269-274; e, in risposta nello stesso volume, un nuovo articolo di Pravadelli, Legitimacy/Change/Power. Is a New Course in Italian Gender Studies Possible? A response to Chiara Saraceno, pp. 275-279.

2. L'intervento di Pravadelli traccia le grandi linee del femminismo italiano della «second wave» nell'ottica del rapporto tra teoria e pratica politica, per dimostrare in che cosa consista, a suo avviso, la specificità del femminismo italiano rispetto a quelli anglosassoni, ma anche quali sono i punti di convergenza. Secondo Pravadelli, negli anni Settanta e Ottanta si è verificata una convergenza tra teoria e pratica, dal momento in cui femministe universitarie e non si incrociavano e incontravano in svariati contesti politici, culturali e sociali. Pravadelli segnala come particolarmente rilevante la traduzione e pubblicazione, nella rivista «Donnawomanfemme», di testi fondamentali del femminismo americano, inglese, francese che hanno alimentato la riflessione e la ricerca nei differenti spazi politici. D'altra parte, sottolinea che nel femminismo italiano i campi di ricerca più frequentati e investiti sono stati la filosofia, la storia, la letteratura e la psicoanalisi, la sociologia, come provano il "pensiero della differenza" che fa riferimento principalmente alle filosofe di Diotima, lo sviluppo di studi femministi della storia (come è il caso per la Società delle Storiche) e della letteratura (Società delle Letterate). 
e visibile di altre realtà istituzionali rispetto agli studi delle donne e/o di genere 3 .

Fino alla metà degli anni Ottanta non esisteva in Italia il dottorato di ricerca, e ancor meno curriculum di studi di genere (io stessa ho scelto di venire in Francia, per iscrivermi a un dottorato di studi femminili a Paris 8Vincennes). Essendo ora docente in tale dottorato (a cui si è aggiunta nel frattempo la dicitura «études de genre») dovrei essere chiaramente favorevole alla creazione accademica di studi di genere ${ }^{4}$. E cosi è. Infatti sono convinta della necessaria esistenza e visibilità di questo spazio di ricerca, anche se nell'ambito dell'università francese ha continuato a lungo a essere quasi ignorato, come pure le numerose ricerche nell'ambito degli studi

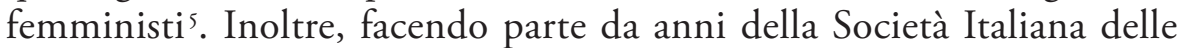
Letterate, la cui appartenenza ha per me un alto valore intellettuale e politico, conosco anche un'altra esperienza del rapporto tra pratica politica, femminismo, pensiero della differenza, e una ricerca sulle problematiche legate a donne, genere, femminismo e differenza sessuale nel campo della letteratura e delle scienze umane e sociali.

Spero di contribuire attraverso questa serie di riflessioni a un racconto del femminismo in cui sono presenti le forme collettive sia nelle attività politiche che nelle produzioni e creazioni culturali, artistiche, letterarie. Pur non avendo nessuna pretesa di darne una visione esauriente — del resto impossibile, vista la diversità e molteplicità degli apporti — cercherò di farne emergere alcuni momenti e aspetti significativi, proprio per pensare la relazione a me cara tra teorie e pratiche poetiche e politiche, nella politica delle donne e di genere, anche tenendo presenti l'evoluzione delle preoccupazioni, delle denominazioni rispetto al movimento delle donne, ai femminismi, all'emergere di altre pratiche e significati nell'ambito dell'accademia, soprattutto degli studi femminili/studi di genere.

La controversia Pravadelli-Saraceno porta sulla valutazione del 'ritardo' dell'istituzionalizzazione degli studi di genere in Italia rispetto alla col-

3. Vari studi esistono sull'argomento: cfr. in particolare $A$ che punto è la storia delle donne in Italia, a cura di Anna Rossi-Doria, Roma, Viella, 2003; Gli studi delle donne in Italia, a cura di Paola Di Cori, Donatella Barazzetti, Roma, Carocci, 20oI; Questioni di teoria femminista, a cura di Paola Bono, Milano, La Tartaruga, 1993; Paola Bono, Esercizi di differenza, Ancona, Milano, Costa \& Nolan, I999; The Lonely Mirror: Italian Perspectives on Feminist Theory, a cura di Paola Bono, Sandra Kemp, London, Routledge, I993.

4. Rinvio alla mia esperienza perché credo sia necessario, almeno nel mio caso, far valere e sottolineare come il dibattito teoria/pratica, istituzione/pratica fuori istituzione attraversi e contrassegni i percorsi di vita e intellettuali in prima persona. Questa affermazione conferma del resto il processo di conoscenza come pratica di una soggettività e un sapere situati, il che rappresenta un contributo essenziale del femminismo.

5. Di fatto solo in questi ultimi anni sono nate varie iniziative per recensire gli studi di genere a livello nazionale; cfr. il recentissimo Recensement CNRS Genre: <https://recherche.genre.cnrs.fr/> (data di ultima consultazione 8 dicembre 20I2). 
locazione accademica dei women/gender/gay e lesbian studies nel mondo anglosassone. Pravadelli lo attribuisce in parte alle posizioni antiaccademiche del femminismo militante più radicale degli anni Settanta, mentre Saraceno contesta questa analisi e sostiene che, pur essendoci una forte resistenza dell'establishment accademico al riconoscimento istituzionale delle ricerche e quindi delle ricercatrici/dei ricercatori in questo campo, molte iniziative sono state appoggiate e si sono sviluppate grazie alla creazione di organismi interni all'università (comitati pari opportunità) il cui compito è precisamente di sviluppare e sostenere le ricerche di genere. Nella risposta a questo testo, Pravadelli appare piuttosto scettica sulla portata e l'efficacia di questa politica di empowerment degli studi di genere, e stima che la marginalità della ricerca, dell'insegnamento e della formazione di genere all'università sia troppo elevata rispetto a quanto si potrebbe auspicare, a parte alcune strutture che hanno consolidato e rinforzato la loro visibilità - come per esempio il CIRSDe (Centro Interdisciplinare di Ricerche e Studi delle Donne) ${ }^{6}$, oppure la comunità filosofica di Diotima a Verona - che però funziona autonomamente e non corrisponde a un centro di ricerca accademico ${ }^{7}$.

Pur nella differenza di valutazioni, questi articoli sembrano tuttavia concordare sull'utilità e necessità di sviluppare pienamente gli studi di genere nelle università e nei centri di ricerca italiani, per colmare la distanza rispetto alla situazione europea e transatlantica. Dato lo spazio disponibile nella rivista, il quadro dell'esistente non è esaustivo in nessuno dei due casi, anche se Pravadelli come Saraceno sottolineano la preminenza di discipline come la filosofia, la letteratura, la sociologia, la storia, la psicoanalisi, la sociologia, gli studi artistici e culturali (cinema, media, arte), mentre scarsi permangono gli apporti a livello nazionale e internazionale nel campo delle scienze.

$\mathrm{Da}$ questo breve riassunto si potrebbe pensare che l'analisi verta più sugli aspetti quantitativi che qualitativi della ricerca, lasciando da parte la discussione sulla valutazione politica dell'istituzionalizzazione degli studi sulle donne/di genere, da cui tra l'altro partiva l'intervento inaugurale di Paola Bono nel convegno del 2009. Questa questione sembra ancora meno attuale e problematica dal momento in cui assistiamo a una crescita e valorizzazione di tali studi, grazie soprattutto all'impulso delle politiche di ricerca europee di genere, e iniziative di censimento delle ricerche esistenti (analoghe al censimento CNRS in Francia).

6. Cfr. il sito internet <www.cirsde.unito.it> (data di ultima consultazione 8 dicembre 20I2).

7. Cfr. <www.diotimafilosofe.it> (data di ultima consultazione 8 dicembre 20I2). 
Questa evoluzione, che non è certo generalizzata, non dovrebbe tuttavia accantonare una riflessione sulle modalità, gli obiettivi, i nodi della teoria/pratica politica riguardo alla produzione di ricerca, di sapere, di pensiero. Anche in questa prospettiva mi preme riprendere alcune considerazioni di Pravadelli, che sottolineava l'importanza del lavoro di traduzione effettuato dalla rivista "DWF», fondata nel 1975 , che ha immesso nel contesto femminista italiano i testi più importanti del pensiero americano ed europeo ${ }^{8}$.

Come i movimenti studenteschi del '68, anche il movimento di liberazione delle donne ha espresso diffidenza o reticenza nei riguardi delle università, percepite come sedi di potere accademico, di "mandarinato", simbolo del sapere/potere patriarcale fallocentrico. Questo in Francia come in Italia (un'eccezione è Vincennes, ma con alcune precisazioni da fare) ${ }^{9}$. Non c'era altra possibilità per una pratica politica che star fuori, altrove. Se avessi seguito fino in fondo questo orientamento del pensiero femminista non avrei mai dovuto fare un dottorato di études féminines, ma appunto il modo e le forme di questo dottorato mi sembravano andare nello stesso senso del movimento delle donne, e non contro, anche se in uno spazio accademico già marginale, spostato rispetto all'accademia, quale era all'epoca Vincennes. La scelta da me fatta alla fine degli anni Settanta, in fondo, si ritrova altrimenti nei collegamenti numerosi e significativi tra ricercatrici, insegnanti, militanti a livello delle riviste. Come vedremo tra poco.

Se ancora pochi sono all'ora attuale i corsi di laurea, master o dottorati di studi femminili o di genere in Italia (ma negli ultimi anni la situazione è cambiata) esistono luoghi e spazi di riflessione molto rilevanti che si avvalgono appunto della collaborazione del mondo universitario (in maggioranza femminile, ma non solo). Questo significa che le riviste di genere non sono sempre nello spazio accademico, ma all'infuori di esso. Tuttavia la mia prima domanda è la seguente: in che modo questo spazio di discussione dell'editoria femminista è meno visibile, e quindi non fa storia o scuola, almeno tanto quanto i seminari e i diplomi universitari? Proprio a un'assemblea della Società Italiana delle Letterate, che si è tenuta a Napoli, Bia Sarasini, giornalista e militante femminista fin dagli anni Settanta, oltre che docente in master di Pari Opportunità, evocava la necessità di pensare la SIL come un'istituzione che è andata crescendo dalla sua nascita

8. Cfr. Veronica Pravadelli, Women and Gender Studies, Italian Style, cit., p. 63.

9. Ci sono stati seminari e assemblee MLF a Paris 8-Vincennes prima ancora che fosse creato il DEA di studi femminili, nel 1974: cfr. Antoinette Fouque, Génération MLF I968-2008, Paris, Editions des femmes, 2008. 
(1995-1996) e che ha organizzato attività seminariali, convegni, atti, libri, riviste, eventi, costituendo man mano un considerevole patrimonio intellettuale, letterario, politico. Assodato il fatto che in Italia esistano poche scuole di studi di genere, ci sono numerose società (le più prestigiose le Società delle Storiche, delle Filosofe, delle Letterate) che offrono da anni scuole estive, convegni, incontri, pubblicazioni.

Certo, le modalità della ricerca (anche a livello finanziario) non sono paragonabili con l'area anglosassone (soprattutto americana). Non parliamo poi in campo editoriale (penso a case editrici universitarie). C'è inoltre il fatto, non secondario, della lingua, l'italiano non è lingua franca a livello scientifico, come l'inglese, e quindi necessita traduzioni, in inglese, in spagnolo o in francese.

Non mi sembra che all'ora attuale esistano studi approfonditi sulle riviste 'femministe' italiane; quelle a cui vorrei rinviare sono piuttosto riviste di riflessione, dialogo, approfondimento, piuttosto che riviste militanti (anche se il risvolto politico è sempre presente con modalità diverse secondo le riviste e i periodi). Come ho già fatto notare, le riviste nascono fuori dalle università, non sono riviste specialistiche né universitarie, non ricevono finanziamenti pubblici, sopravvivono grazie agli abbonamenti. Anche se molte delle autrici appartengono al mondo universitario, al momento in cui vi scrivono l'appartenenza militante e politica prevale sul discorso più precisamente scientifico e istituzionale. Questo rovesciamento e questa articolazione di linguaggi differenti attraversano, spesso, le stesse protagoniste. Il che mi sembra un aspetto importante.

In un momento come quello attuale in cui la valorizzazione della ricerca (analogamente a quanto avviene con l'AERES in Francia) opera uno scarto di tutta la produzione scientifica che non sia strettamente attinente a un numero di pubblicazioni designato su criteri certo validi ma strettamente disciplinari, lo scarto tra le pratiche di ricerca diventa molto più importante e rigetta fuori dall'istituzione scientifica e universitaria le ricerche sulle discipline associate alla riflessione sulle politiche di genere.

Negli anni Settanta creare riviste femministe, contribuire con scritti e traduzioni era una scelta consapevole e militante, in nessun modo le collaborazioni a queste riviste si ponevano come contributo al discorso universitario, anche se spesso gli scritti erano frutto di ricerche di grande qualità scientifica. La figura delle donne si ritrovava in quella dell'outsider, nell'estraneità voluta, assunta, a una struttura di genere (il neutro maschile) da cui la pratica militante si era dissociata.

Non per niente nella fase attuale di apparente recessione dalla politica e della politica (ma di riflussi e di backlash ce ne sono già stati vari) 
la ricerca e la riflessione hanno acquistato maggior importanza, anche se non mancano altre pratiche, meno visibili o altrimenti visibili (siti web, performance, eventi spettacolari pubblici).

$\grave{E}$ evidente che il far ricerca, lo studio, la pubblicazione valorizzano e diffondono un patrimonio di pensieri e di scritti che spesso rischiano l'ignoranza o la dimenticanza. Questo è vero per le opere di letteratura, le opere artistiche, tutte le forme di produzione e azione. Indubbiamente, le riviste e le pubblicazioni di ricerche hanno svolto un immenso lavoro di trasmissione, interpretazione, valorizzazione, indipendentemente dall'appartenenza o meno all'accademia. E anzi, realizzando in modo estremamente creativo quell'indipendenza intellettuale che Virginia Woolf auspicava nelle famose Tre ghinee: non si può pensare, politicamente, vivere in questo mondo, senza la possibilità di accedere al sapere, all'educazione, a parole e azioni libere, indipendenti; in particolare per le donne, che nel sistema patriarcale hanno vissuto nella dipendenza dal maschile. Le riviste coltivano quindi questa autonomia, questa indipendenza dalle sovvenzioni regionali, nazionali, universitarie, e vivono grazie alla comunità delle lettrici e lettori (il che non esclude forme di mecenatismo).

Se mettiamo insieme tutti questi dati con ben altri che si dovrebbero raccogliere e valutare si possono capire certe conclusioni su una situazione di marginalità (con eccezioni, certo) rispetto ad aree molto più dense, più vistose e frequentate. Una conseguenza per esempio del tutto banale: se si vogliono fare leggere i libri decisivi di pensatrici come Carla Lonzi ${ }^{\mathrm{IO}}$, Adriana Cavarero ${ }^{\mathrm{II}}$, le filosofe di Diotima ${ }^{\mathrm{I2}}$ bisogna ricorrere a traduzioni dei libri (quando esistono) in inglese o in spagnolo, meno in francese. Potrei dire che, al contrario, in quanto italiana e di formazione piuttosto anglista, ho imparato il francese tardivamente per poter accedere ai testi e pensieri che mi appassionavano.

Io. Carla Lonzi è considerata come una figura di riferimento fondamentale per il femminismo italiano. Tra le sue opere: Sputiamo su Hegel, La donna clitoridea e la donna vaginale e altri scritti, Milano, Scritti di rivolta femminile, 1978 .

II. Adriana Cavarero è tra le fondatrici di Diotima e ha partecipato a vari volumi pubblicati dal gruppo, tra cui: Diotima, Il pensiero della differenza sessuale, Milano, La Tartaruga, 1987; Mettere al mondo il mondo, Milano, La Tartaruga, I990. È anche autrice di numerosi volumi, tra cui: Nonostante Platone. Figure femminili nella filosofia antica, Verona, ombre corte, 2009 (prima edizione 1990); Tu che mi guardi, tu che mi racconti, Milano, Feltrinelli, 1997, sulla filosofia della narrazione; $A$ più voci, Milano, Feltrinelli, 2003, sulla filosofia dell'espressione vocale; fino al più recente Orrorismo ovvero la violenza sull'inerme, Milano, Feltrinelli, 2007.

I2. Più di una decina sono attualmente i volumi pubblicati di Diotima. Ogni volume nasce dalla problematica discussa e approfondita nel grande seminario annuale della comunità filosofica che si tiene nei pressi di Verona: oltre a quelli citati nella nota precedente: Luisa Muraro, L'ordine simbolico della madre, Roma, Editori Riuniti, 199I; Diotima, Il cielo stellato sopra di noi, Milano, La Tartaruga, I992; L'ombra della madre, Napoli, Liguori, 2007; i numerosi volumi sull'autorità femminile e l'affidamento, fra cui Oltre l'ugualianza, le radici femminili dell'autorità, Napoli, Liguori, 1995 . 
Le studentesse attuali del nostro master di genere, Genre(s), pensées des différences, rapports de sexes, non solo vogliono effettuare una formazione universitaria, ma hanno un riferimento politico più o meno definito per riuscire a leggere realtà, mondi, società in cui la questione dell'essere donna o uomo, l'esistenza di disuguaglianze, discriminazioni, legislazioni non paritarie, la questione della sessualità e delle sessualità non sono affrontate. Esiste nondimeno, ed è più diffuso di quanto si può pensare, il bisogno di riflettere, di trovare strumenti di ricerca e orientamenti all'altezza di tali problemi, della loro molteplicità e complessità.

Vorrei tornare ora un poco indietro su quello che affermava e proponeva Bia Sarasini: in primo luogo, in cosa consiste questo patrimonio di saperi, pratiche, produzioni? In secondo luogo, come valorizzarlo? In quanto c'è, ma non è usato, utilizzato, trasmesso quanto dovrebbe esserlo.

Dapprima, bisognerebbe connettere una politica della trasmissione a una politica della traduzione di testi, pensieri, contesti culturali, storici, sociali. Vorrei dare alcuni esempi significativi che tutti coglieranno: il famoso saggio di Hélène Cixous, Le rire de la Méduse, che è stato appena ripubblicato dalle edizioni Galilée con il titolo Le rire de la Méduse et d'autres ironies, era stato pubblicato inizialmente sul numero 6I della rivista "L'Arc», rivista affatto femminista, ma essendo il 1975 quel numero era dedicato a Simone de Beauvoir et la lutte des femmes. In seguito, il successo del saggio in tutto il mondo fu dovuto (a parte i suoi meriti intrinsechi) alle traduzioni inglesi, la prima nella rivista "Signs» (I976), poi nel famoso volume di Elaine Marks e Isabelle de Courtivron New French Feminisms, quindi in una serie di Readers (almeno quattro). In Italia è stato tradotto soltanto nel 1997 e pubblicato in un volume a più voci, Critiche femministe e teorie letterarie (Bologna, CLUEB). Altri testi della stessa scrittrice erano stati pubblicati in precedenza, per esempio in «DWF» e «Leggendaria» (per mia cura) ${ }^{\mathrm{I}}$.

Certo, le situazioni sono incomparabili, se non altro a livello del lettorato universitario, e il fatto che le edizioni universitarie siano reticenti a pubblicare testi e saggi sulle tematiche di studi di genere non facilita la loro diffusione (contrariamente a quanto succede nel mondo universitario nordamericano).

Tuttavia esistono altri spazi in Italia che mantengono, in questi anni difficili, una rete di comunicazioni, incontri, recensioni, diffusioni molto

13. È da segnalare che proprio questa rivista, «DWF», ha tradotto e pubblicato a varie riprese testi importanti del femminismo internazionale, per esempio Adrienne Rich, Eterosessualità obbligatoria ed esistenza lesbica, in Amore proibito. Ricerche americane sull'esistenza lesbica, "Nuova DWF", 23-24, gennaio 1985, pp. 5-40. 
fitta. Riviste come «DWF», «Leggendaria», «Via Dogana», «Leggere donna» non solo pubblicano articoli e saggi su temi e dibattiti, ma informano sugli eventi, manifestazioni, incontri, seminari a livello nazionale e talvolta internazionale. E negli ultimi anni la creazione dei siti aggiunge alla rivista cartacea la rivista online con funzioni supplementari (blog, archivi, comunicazione con autrici e redattrici). Questi aspetti mi sembra non siano presenti nelle riviste strettamente universitarie e di ricerca, e interessino aree di lettura diverse, più ampie, più sensibili politicamente.

Perché le riviste femministe di indirizzo letterario o storico o scientifico non hanno in Italia (ma anche in Francia) pari notorietà e influenza che altre grandi riviste 'storiche'? Anche in questo campo dell'editoria vige forse una forma di marginalizzazione rispetto alle istituzioni letterarie, o altre, dovuta in parte alla (almeno) doppia valenza, politica e scientifica, di queste pubblicazioni. Infatti è indubbio che riviste come «DWF», "Leggendaria», "Via Dogana» nascono da un desiderio politico e di politica, e nel contempo da un pensiero delle pratiche, dei saperi molteplici che convocano le competenze e le ricerche, i pensieri come gli scritti di alto valore. Quindi certo si può attribuire questa squalifica alla marginalizzazione, ma anche a una resistenza a una certa forma di discorso, di pubblicazione, che si mantiene nell'ambito della "comunità» di pensiero e pratiche politiche, almeno all'inizio del movimento femminista e delle donne. Questo senso e/o pratica della politica viene a definirsi nel corso degli anni e di varie generazioni di donne e studiose (alcuni studiosi) mantenendo una coerenza di fondo, e rivelando capacità di crescita, allargamento, accoglimento di altre e diverse pratiche politiche.

Ma sono necessarie alcune osservazioni più specifiche sulla presentazione di queste riviste. "Donnawomanfemme» ("DWF») a lungo con il sottotitolo Quaderni di studi internazionali sulla donna (questo sottotitolo è ora stato tolto). Nel numero di «DWF» del I988, Responsabilità politica (riflessione midterm), l'orizzonte e gli scopi mi sembrano ben delineati fin dal titolo, che traduce in modo molto significativo uno degli obiettivi della creazione e pubblicazione di questa rivista, che ha conosciuto varie versioni e vite (la prima è del 1975-1976), rispetto al senso dell'istituzione. Vi sono presenti snodi importanti di riflessione, che insistono sull'articolazione teoria/pratiche, singolarità/comunità, affettività/politica:

I4. Il sito attuale, con possibilità di visionare l'archivio, è <www.dwf.it> (data di ultima consultazione 8 dicembre 2012). 
Il sentimento di appartenenza al genere politico femminile è — per natura del vincolo - «sentimento politico». Si fonda (e fonda) relazioni significative tra donne che di quel sentimento sono i soggetti politici [...]. Che la vita sia il riferimento per misurare il rapporto tra la produzione teorica e la pratica politica che questa si propone di mettere in atto è stato - e noi riteniamo possa ancora essere - il criterio «scientifico» per poter sostenere politicamente un uso non moderato delle ipotesi. L'ancoraggio continuo al vissuto quotidiano ci ha consentito in passato di elaborare ipotesi apparentemente utopiche e di imporle come politiche. La separazione tra vita e politica dà luogo invece a misure che implicano la moderazione, sia della teoria che del comportamento. (p. 5)

È chiaro che ogni rivista, pur aspirando a uno spazio editoriale riconoscibile e a un'identità propria ${ }^{\mathrm{I}}$, è nel contempo spazio aperto di dibattito che si arricchisce di volta in volta di numerosi contributi irriducibili a un discorso univoco e compatto. Piuttosto emerge man mano una rete di soggetti, sia a livello tematico che di voci che intervengono con i loro scritti, competenze e posizioni. E, fatto più evidente e rilevante, il lavoro redazionale è frutto collettivo, orientato da direttrici e gruppo redazionale.

Questa forma di pubblicazione ha quindi una rilevanza politica e teorica estremamente importante, perché sottolinea proprio una delle caratteristiche dei movimenti: l'insieme delle singolarità in rete, l'irriducibilità a un pensiero monologante e uninominale, senza peraltro scadere nella frammentarietà, dal momento che ogni volta si tratta di sviluppare discorsi e linee di pensiero con cui poter interferire e interloquire. Non esistono forse, all'ora attuale, studi sulla politica del nome collettivo, sulla polifonia o polinomia, che non significa cancellazione dell'individualità ma iscrizione in uno spettro plurivoco di dibattito, di pensieri e idee.

"Leggendaria» ${ }^{16}$ mantiene fino a tutt'oggi il sottotitolo Libri Letture Linguaggi, sottotitolo che dice molto chiaramente la sua linea editoriale principale: l'aggiornamento su pubblicazioni di vario tipo, saggistico, romanzesco, sia in ambito italiano che internazionale riguardanti le donne, il genere, il femminile. La parte centrale della rivista ospita in ogni numero il tema con differenti articoli o interviste, e significativamente gli articoli si appoggiano su una bibliografia scelta di pubblicazioni anche in lingua originale, oppure sono vere e proprie recensioni di libri usciti di recente. «Leggendaria» offre inoltre una presentazione grafica molto curata e varia,

I5. In cui si ritrova il gruppo redazionale che la crea, anche se la composizione di questo gruppo può variare negli anni. Tra l'altro la redazione della rivista, con le persone, in maggioranza donne, che vi collaborano, costruisce nel corso degli anni una comunità importante, senza parlare del lettorato (abbonamenti e acquisti in libreria).

I6. L'indirizzo del sito è <www.leggendaria.it> (data di ultima consultazione 8 dicembre 20I2). 
inserendo in ogni numero uno striscione (fotografia, disegno, scultura, pittura) ispirato all'opera di un artista (femminile o maschile). Non semplice illustrazione ma proposta di altra lettura in parallelo. Di fatto, l'arte moderna e contemporanea è estremamente presente. Lo stesso dicasi per il teatro, il cinema, anche se più saltuariamente. La lettura sul sito della pagina Chi siamo ci dà dei segni importanti delle linee direzionali e progettuali della rivista, che mi interessano particolarmente in questa sede:

La rivista si colloca nell'ambito degli strumenti del pensiero critico, assumendo il pensiero delle donne come punto di vista collocato e privilegiato per la critica del presente: ha un approccio multidisciplinare e multiculturale rivolto a uomini e donne ed è particolarmente attenta alla trasmissione generazionale. ${ }^{17}$

Lindice dei primi venti numeri della rivista, allegato al primo numero del 2000 (curato da Giuliana Misserville), ci offre un panorama molto interessante dei temi e dei titoli della rivista. Se la ricchezza degli apporti (recensioni, saggi tematici) sulla letteratura e la politica italiana conferma un orientamento maggiore della politica editoriale, bisogna tuttavia rilevare una grandissima attenzione all'internazionale: numerosi fascicoli monografici sono dedicati a vari paesi nel mondo, accostando in permanenza scenari politici e storici e voci letterarie e artistiche di donne in lotta: l'Algeria (I4, 1999), l'Africa (7-8B, I998), l'Argentina (8, 1999), l'India (13, 1999), il Perù $(63-64,2007)$ le Italiane d'America $(45-46,2004)$ le Afroamericane $(20,2000)$ eccetera. Ma al centro dei diversi numeri sono anche alcune città italiane - Torino, Genova, o L'Aquila (numero speciale dedicato a Terre mutate, dopo il terremoto del 2009). Una cartografia in cui l'attenzione alla cultura letteraria femminile di lingua italiana si trova in permanenza spostata e ravvicinata a opere, autrici, eventi del contesto mondiale, dando luogo a una bibliografia di genere di un'estrema ricchezza.

Per quanto riguarda il trimestrale $«$ Via Dogana» ${ }^{18}$ risalta subito, fin dal primissimo numero (maggio 1983), un'insistenza sul politico, a partire dal sottotitolo significativo Rivista di pratica politica, mentre in un quadratino in basso a destra della copertina l'indirizzo di pubblicazione vale come casa editrice, cioè "Libreria delle donne» ${ }^{19}$ : le lotte delle donne,

I7. Dall'editoriale online.

18. In <www.libreriadelledonne.it> (data di ultima consultazione 8 dicembre 20I2).

19. È noto che la Libreria delle donne di Milano è uno dei luoghi più conosciuti di pensiero e pratica politica di quello che si denomina come il pensiero della differenza, che vede, accanto ai nomi di Luisa Muraro, Lia Cigarini, Annarosa Buttarelli, molte altre che appartengono a Diotima o ad altri gruppi, pensatrici, scrittrici, saggiste, donne (e alcuni uomini) in politica. 
un rapporto conflittuale con la cultura (identificata come dominata dal maschile), tuttavia un interesse vivissimo per i libri, «perché i libri sono legati ad altre cose che c'interessano quanto e più dei libri ${ }^{20}{ }^{\circ}$

Nei libri, come in altre cose dette o fatte da donne - convegni, riviste, musica, cinema, ecc. - cerchiamo i punti di rispondenza con noi. Scoprire tale rispondenza ci dà la forza della conferma. Di quelle donne eccezionali che riescono a mettersi in rapporto con il mondo in maniera originale, vogliamo fare le nostri madri. Anche quando ci capiterà di criticarle, sarà solo per farle nostre. (I, 1983)

Il titolo ci dà anche una chiave importante del rapporto alla cultura e alla lettura collegato a una genealogia femminile 'qualcuna' e a quella che sarà una delle nozioni più importanti e discusse della Libreria di Milano: l'autorità femminile, l'affidamento, l'ordine simbolico della madre. «Non viene spontaneo fare riferimento alle parole di una propria simile. Arrivarci costituisce forse il passo più importante della nostra politica.» Come si vede non è solo un riferimento culturale, o scientifico, una citazione o una memoria: è un posizionamento critico che permette il riconoscimento e l'appropriazione di una ricchezza che non è più sotto il segno di un universale neutro, ma di una possibile differenza sessuale di parte femminile.

Mi sembra che una lettura di queste riviste attraverso gli anni, un'analisi che tenga conto del corso della storia, degli eventi e delle politiche, potrebbe restituire quella parte della storia del pensiero femminile, della cultura di genere forse sotterranea o altrimenti visibile e leggibile, proprio perché si muove negli interstizi, tra siti accademici che stentano a riconoscere il valore delle ricerche e delle presenze femminili (comprese le varie evoluzioni teoriche) e spazi molto più fluidi anche rispetto ai media, ma dove sono attive da anni un numero importante, per quantità e qualità, di attrici, e qualche attore capaci di analizzare, interpretare oltre che informare e orientare la lettura della realtà postmoderna e globale, di affinare la lettura di genere in tutte le sue valenze politiche, storiche, culturali. 